\title{
Adaptive Multi-GPU Exchange Monte Carlo for the 3D Random Field Ising Model
}

\author{
Cristóbal A. Navarro ${ }^{\mathrm{a}, *}$, Wei Huang ${ }^{\mathrm{b}}$, Youjin Deng ${ }^{\mathrm{b}, \mathrm{c}}$ \\ ${ }^{a}$ Instituto de Informatica, Universidad Austral de Chile, Valdivia, Chile. \\ ${ }^{b}$ Hefei National Laboratory for Physical Sciences at Microscale, Department of Modern Physics, University of \\ Science and Technology of China, Hefei, 230027, China. \\ ${ }^{c}$ State Key Laboratory of Theoretical Physics, Institute of Theoretical Physics, Chinese Academy of Sciences, \\ Beijing 100190, China.
}

\begin{abstract}
This work presents an adaptive multi-GPU Exchange Monte Carlo approach for the simulation of the 3D Random Field Ising Model (RFIM). The design is based on a two-level parallelization. The first level, spin-level parallelism, maps the parallel computation as optimal 3D thread-blocks that simulate blocks of spins in shared memory with minimal halo surface, assuming a constant block volume. The second level, replica-level parallelism, uses multi-GPU computation to handle the simulation of an ensemble of replicas. CUDA's concurrent kernel execution feature is used in order to fill the occupancy of each GPU with many replicas, providing a performance boost that is more notorious at the smallest values of $L$. In addition to the two-level parallel design, the work proposes an adaptive multi-GPU approach that dynamically builds a proper temperature set free of exchange bottlenecks. The strategy is based on mid-point insertions at the temperature gaps where the exchange rate is most compromised. The extra work generated by the insertions is balanced across the GPUs independently of where the mid-point insertions were performed. Performance results show that spin-level performance is approximately two orders of magnitude faster than a singlecore CPU version and one order of magnitude faster than a parallel multi-core CPU version running on 16-cores. Multi-GPU performance is highly convenient under a weak scaling setting, reaching up to $99 \%$ efficiency as long as the number of GPUs and $L$ increase together. The combination of the adaptive approach with the parallel multi-GPU design has extended our possibilities of simulation to sizes of $L=32,64$ for a workstation with two GPUs. Sizes beyond $L=64$ can eventually be studied using larger multi-GPU systems.
\end{abstract}

Keywords: GPU Computing, Adaptive Temperatures, Exchange Monte Carlo Algorithm, Random Field Ising Model

\section{Introduction}

Monte Carlo methods have become a convenient strategy for simulating finite size spin lattices towards equilibrium and for measuring their average physical observables. Classic spin models such as Ising [1, 2] and Potts [3] have usually been simulated with the MetropolisHastings algorithm $[4,5]$, cluster $[6,7,8]$ or worm algorithm [9], with the last two being

\footnotetext{
* Corresponding author

Email address: cristobal.navarro.g@gmail.com (Cristóbal A. Navarro)
}

Preprint submitted to Journal of Computational Physics

April 12, 2016

(C) 2016. This manuscript version is made available under the Elsevier user license http://www.elsevier.com/open-access/userlicense/1.0/ 
more efficient than the first at reaching equilibrium near the critical temperature $T_{c}[10]$. The model of interest for this work is the bimodal 3D Random Field Ising Model (RFIM), which is one of the archetypal disordered systems. The RFIM defines the following Hamiltonian

$$
\mathcal{H}=-J \sum_{\langle i, j\rangle} s_{i} s_{j}-h \sum_{i} h_{i} s_{i}
$$

where $J>0$ is the ferromagnetic interaction constant between nearest neighbors $\langle i, j\rangle, s_{i}, s_{j}$ correspond to Ising spins, $h$ is the strength of the random field and $h_{i}= \pm 1$ are independent quenched random variables that take values from a bimodal distribution of the form

$$
P\left(h_{i}\right)=\frac{1}{2}\left[\delta\left(h_{i}-1\right)+\delta\left(h_{i}+1\right)\right] .
$$

Other random field probability distributions have been considered and studied, such as Gaussian and very recently the tri-modal distribution $[11,12,13]$. The competing nature between the interactions of standard Ising model and random fields creates rich physics and has challenged physicists for several decades. For the 3D RFIM, there is an ordered ferromagnetic phase at low temperature for small enough field strength [14]. However, the properties of phases and the nature of phase transitions are still controversial. What makes the 3D RFIM interesting and challenging is the fact that its energy landscape is highly degenerated and complex due to the quenched disorder introduced by the random field. Classic Markov Chain Monte Carlo (MCMC) algorithms are no longer effective at simulating these systems, since they have difficulties at overcoming the many local minima found in the energy landscape. This simulation problem typically occurs at the low temperature regime, i.e., $T \leq T_{c}$, which is a region of great interest.

Instead of simulating the system using one instance of the lattice, as in a classic MCMC approach, it is possible to simulate $R$ replicas at different temperatures and exchange information among them with a certain probability. It has been shown that successive repetitions of alternating simulation and exchange can eventually overcome the local minimum trap problem $[15,16]$. The algorithm based on this principle is known as the Exchange Monte Carlo method, or Parallel Tempering, and it is one of the most used algorithms for simulating systems with quenched disorder. The notion of the algorithm was first introduced by Swendsen and Wang in 1986 [16] and then extended by Geyer in 1991 [17]. Hukushima and Nemoto presented in 1996 the full method as it is known today [15].

The replica based approach has been used in the past to simulate 2D and 3D spin glass models $[15,18,19,20,21]$. While it is true that replica based methods can overcome the main difficulty of the Monte Carlo simulation in disordered systems, the computational cost is still considered a problem, since it requires at least $\Omega\left(R L^{d}\right)$ operations per exchange step, with $L$ being the linear size of the lattice and $d$ the number of dimensions of the system. In this work we are interested in the particular case of simulating the bimodal 3D RFIM using the Exchange Monte Carlo method adapted to massively parallel architectures such as the GPU.

The fast evolution of parallel architectures [22] such as multi-core CPUs and modern GPUs provides an opportunity to further improve the running time of Monte Carlo simulation. Although Parallel Tempering (PT) is a pleasingly parallel algorithm that is as suitable for CPUs as for GPUs, we are interested in exploiting the benefits that modern GPUs can provide in terms of performance. The GPU architecture, being a massively parallel processor, can 
offer up to ten times the FLOPS and memory bandwidth of modern multi-core CPUs. But in order to obtain such benefits of performance and efficiency, GPU-based algorithms need to be carefully designed and implemented to fulfill the restrictions of the GPU architecture. This computational challenge, combined with the physical motivation of studying the bimodal 3D RFIM, constitute the main motivations of our work.

This work presents an adaptive multi-GPU approach for the simulation of the $3 D$ Random Field Ising Model. The implementation uses two levels of parallelism; (1) spin parallelism that scales in the presence of faster GPUs, and (2) replica parallelism that scales in the presence of multiple GPUs. Both levels, when combined together, provide a substantial boost in performance that allows the study of problems that were too large in the past for a conventional CPU implementation, such as $L=64$. In addition to the parallelization strategy, we also propose a temperature selection scheme based on recursive insertions of temperature points to improve the exchange rate at the regions where it is often less frequent. Physical results have been included for the $3 \mathrm{D}$ random field model at sizes $L=\{8,16,32,64\}$ using a field strength of $h=1$.

The rest of the paper is organized as follows: Section 2 presents the related works regarding parallel implementations of the Exchange Monte Carlo method. In Sections 3 and 4 the levels of parallelism and the adaptive temperatures strategy are explained in detail. Sections 5,6 and 7 give technical details about the implementation, analyze the performance results of the implementation as well as test the quality of the adaptive temperatures strategy. Sections 8 and 9 present physical observables for $L=\{8,16,32,64\}$ and comment on the technical possibilities of reaching sizes of $L=128$ and beyond. Finally, Section 10 concludes and discusses the work.

\section{Related Work}

Several works have shown the benefits of GPU-based implementations of MCMC algorithms for spin systems. The Metropolis-Hastings algorithm has been efficiently re-designed as a GPU algorithm for both 2D and 3D lattices [23, 24, 25, 26]. The parallelization strategy is usually based on the checkerboard decomposition of the problem domain, where black and white spins are simulated in a two-step parallel computation. Although the checkerboard method violates detailed balance, it still obeys the global balance condition which is sufficient to ensure convergence of the stochastic process. M. Weigel proposed the double checkerboard strategy, that takes advantage of the GPU's shared memory [27, 28, 29] for doing partial Metropolis sweeps entirely in cache. In the work of Lulli et. al. [26] the authors propose a highly optimized single and multi-GPU schemes that reorganize the lattice in alternating slices in order to achieve efficient memory access patterns.

For cluster algorithms, recent works have proposed single and multiple GPU implementations, for both Ising and Potts models [30, 31, 32, 33]. For the case of the Swendsen and Wang algorithm, which is a multi-cluster one, some use a parallel labeling strategy based on the work of Hawick et. al. [34]. The cluster work of Weigel uses an approach based on self-labeling with hierarchical sewing and label relaxation [33]. A study on the parallelism of the Worm algorithm has been reported by Delgado et al. [35]. The authors conclude that an efficient GPU parallelization is indeed hard because very few worms stay alive at a given time.

Two GPU-based implementations of the Exchange Monte Carlo method have become important in the literature. One was proposed by Weigel for the 2D Edwards-Anderson Spin 
Glass [28], in which the author treats all the replicas of the system as one large lattice, therefore additional replicas in practice turn out to be additional thread blocks. The second work is by Ye Fang et. al [36] and they propose a fast multi-GPU implementation for studying the 3D Spin Glass. In their work, the authors propose to keep the replicas in shared memory instead of global memory. This modification provides a performance in memory accesses that can be up to an order of magnitude faster than global memory ones, but limits the size of the lattice to the amount of shared memory available, which for todays GPUs it means 3D lattices of size $L \leq 16$. Katzgraber et. al. proposed a method for improving the temperature set in the Exchange Monte Carlo method [37]. The strategy is based on keeping a histogram record of the number of round trips of each replica (i.e., the number of times a replica travels from $T_{\min }$ to $T_{\max }$ and vice versa). Once the histogram is built, the temperature set can be re-arranged, bringing pairs of replicas with low exchange rate closer together in temperature, increasing their exchange rate. Another strategy was presented by Bittner et. al., where they propose a method for obtaining a good set of temperatures and also they propose to set the number of lattice sweeps according to the auto-correlation time observed [38].

To the best of our knowledge, there is still room for additional improvements regarding GPU implementations for the Exchange Monte Carlo method, such as using concurrent kernel launches, extending the double checkerboard strategy to 3D, optimal 3D thread blocks, globalmemory multi-GPU partitions, and low level optimizations for the case of the 3D RFIM, among others. In relation to choosing the temperature set, it is still possible to explore different adaptive strategies based on recursive algorithms. In the next section we present our parallel implementation of the Exchange Monte Carlo method, which includes all these improvements recently mentioned, as well as an adaptive strategy for choosing an efficient temperature set.

\section{Multi-GPU approach}

For a multi-GPU approach, we analyze the Exchange Monte Carlo to find out how many levels of parallelism exist.

\subsection{Parallelism in the Exchange Monte Carlo method}

The Exchange Monte Carlo method is an algorithm for simulating systems with quenched disorder. The notion of exchanging replicas was first introduced by Swendsen and Wang in 1986 [16], later extended by Geyer in 1991 [17]. In 1996, Hukushima and Nemoto formulated the algorithm as is it known today [15]. The algorithm has become widely known for its efficiency at simulating Spin Glass models and for its simplicity in its definition. The algorithm can be applied with no difficulties to other disordered models as well such as the Random Field Ising Model (RFIM), which is the model of interest in the present work.

The algorithm works with $R$ replicas $\mathcal{X}=\left\{X_{1}, X_{2}, \ldots, X_{R}\right\}$ of the system with each one at a different temperature. The main steps for one disorder realization of the Exchange Monte Carlo algorithm, for the case of the Ising Random Field model, are the following:

1. Choose $R$ different temperatures $\left\{\beta_{1}, \beta_{2}, \ldots, \beta_{R}\right\}$ with $\beta_{i}=1 / T_{i}$ and $\beta_{i}>\beta_{i+1}$, i.e., $T_{i}<T_{i+1}$.

2. Choose an arbitrary random magnetic field $H=\left\{h_{1}, h_{2}, h_{3} \ldots, h_{|V|}\right\}$ with $h_{i}=\operatorname{rand}( \pm 1)$. This instance $H$ of disorder is used for the entire simulation by all $R$ replicas. 
3. Set an arbitrary spin configuration to each one of the $R$ replicas and assign the corresponding temperature, i.e., $X_{i} \leftarrow T_{i}$.

4. [Parallel] Simulate each replica simultaneously and independently in the Random Field Model for $p$ parallel tempering moves, using $H$ for all replicas and a highly parallel MCMC algorithm such as Metropolis-Hastings. At each parallel tempering move, exchange the odd xor even configurations $X_{i}$ with their next neighbor $X_{i+1}$, with a probability given by the transition matrix $W$ :

$$
W\left(X_{i}, \beta_{i} \mid X_{i+1}, \beta_{i+1}\right)= \begin{cases}1 & \text { for } \Delta<0 \\ e^{-\Delta} & \text { for } \Delta>0\end{cases}
$$

where $\Delta=\left(\beta_{i+1}-\beta_{i}\right)\left(\mathcal{H}\left(X_{i}\right)-\mathcal{H}\left(X_{i+1}\right)\right)$. Choosing odd or even depends if the $j$-th exchange is odd or even, respectively.

The algorithm itself is inherently data-parallel for step (4) and provides a sufficient number of data elements for a GPU implementation. In fact, there are two levels of parallelism that can be exploited; (1) spin parallelism and (2) replica level parallelism. In spin parallelism the challenge is to come up with a classic MCMC method that can take full advantage of the GPU parallel power. For this, we use a GPU-based Metropolis-Hastings implementation optimized for 3D lattices. For (2), the problem is pleasingly parallel for a multi-GPU implementation except for the exchange part which could degrade the efficiency of the parallel method. For this, it is of interest to study how well can multi-GPU parallelism scale when varying $R$ and $L$, as well as analyze the scaling in terms of the asymptotic behavior of the parallel and sequential work involved in the exchange phase. Figure 1 illustrates the two levels of parallelism and their organization.

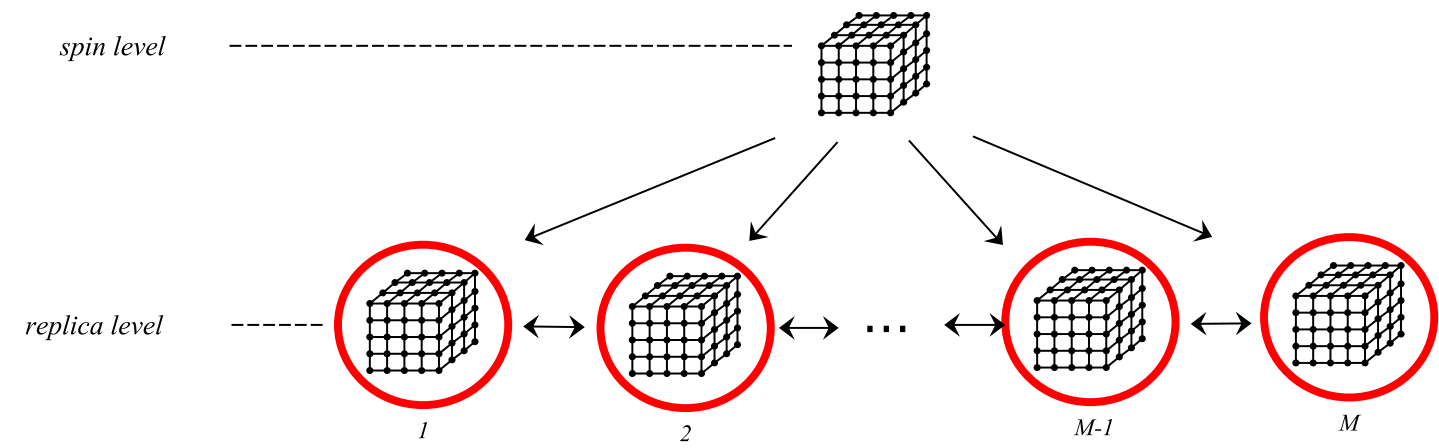

Figure 1: The two levels of parallelization available in the exchange Monte Carlo method.

\subsection{Spin Level Parallelism}

Spin level parallelism corresponds to the parallel simulation of the spins of a single replica. This level is handled with a single CUDA kernel based on the double checkerboard idea proposed by M. Weigel $[29,28]$, which was originally used for $2 \mathrm{D}$ problems, but it can be extended to 3D as illustrated in Figure 2. 


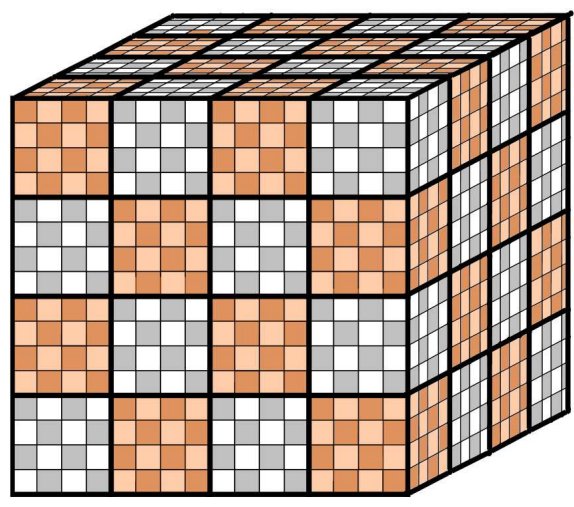

Figure 2: Double 3D checkerboard.

\subsubsection{Structure of the Double 3D Checkerboard}

In order to create a double $3 D$ checkerboard, one can use the fact that it can be built by stacking alternated 2D checkerboard layers over a third dimension. For a given point $p=(x, y, z)$ in $3 \mathrm{D}$ discrete space, its alternation value $A(p)$ is

$$
A(p)=(x+y+z) \quad \bmod 2
$$

A double $3 D$ checkerboard organizes the lattice as several small checkerboards of size $\left|B^{s}\right|=B_{x}^{s} \times B_{y}^{s} \times B_{z}^{s}$, which are again organized as a coarse checkerboard of $\left|B^{c}\right|=B_{x}^{c} \times B_{y}^{c} \times B_{z}^{c}$. A Metropolis-Hastings method based on this approach must alternate the simulation between the checkerboard colors of both the coarse and fine grained tiles, recursively in the two levels of the checkerboard. As a result, we have that one full Metropolis-Hastings sweep consists of four synchronization barriers; two for the fine-grained checkerboard which are local and done inside a single kernel, and two for the coarse-grained checkerboard which are global, performed with two kernels launched one after another. Given that a simple checkerboard simulation only needs half the number of threads, a space of computation of $L^{3} / 4$ threads is necessary and sufficient for maximum parallelism in the double $3 \mathrm{D}$ checkerboard, matching the limit of four synchronization barriers; i.e., $L^{3} /\left(\left(\left|B^{s}\right| / 2\right)\left(\left|B^{c}\right| / 2\right)\right)=4$.

The main advantage of the double checkerboard approach is that spins can be flipped in shared memory space, which is up to two orders of magnitude faster than flipping in global memory space. The block of shared memory consists of a block of spins for simulation plus a 3D halo of spins which are not simulated but are required for the simulation of the spins in the boundary of the block. Given a shared memory block $B^{s}=\left(B_{x}^{s}, B_{y}^{s}, B_{z}^{s}\right)$, its corresponding thread block is $B^{t}=\left(B_{x}^{t}, B_{y}^{t} / 2, B_{z}^{t}\right)$ and has half the number of spins in $B^{s}$. Loading the block of spins into shared memory takes two memory accesses per thread. Loading the halo contributes to additional memory accesses by the boundary threads of $B^{t}$. Once the data is loaded, the checkerboard computation is performed in parallel first for the black spins, then for the white ones, giving a total of two time steps. Finally, once the sweep is finished, the block of spins can be copied back to global memory.

\subsubsection{Coalesced Memory Access}

It is possible to achieve coalesced memory accesses when copying from global to shared and vice versa. To accomplish this, one has to realize that reading and writing the global 
memory does not necessarily need to be done with the checkerboard thread map, but instead can be done using the following map

$$
f\left(t_{x}, t_{y}, t_{z}\right)=\left(t_{x}, t_{y}+\delta, t_{z}\right)
$$

which is fully coalesced when $B_{x}^{s}$ is chosen as a multiple of the warp, currently 32 for modern architectures, with $t=\left(t_{x}, t_{y}, t_{z}\right)$ being the thread in global 3D space and $\delta$ an offset value, 0 or $B_{y}^{s} / 2$, to read the first or second spin, respectively, that corresponds to each thread. The lattice in global memory is organized as consecutive $X Y$ planes, with each plane following a row-major order scheme. Once the data has been loading into shared memory, threads can then be re-mapped with the following $3 \mathrm{D}$ checkerboard map

$$
c\left(t_{x}^{\prime}, t_{y}^{\prime}, t_{z}^{\prime}\right)=\left(t_{x}^{\prime}, \delta+a \cdot\left(t_{y}^{\prime}+t_{x}^{\prime}+t_{z}^{\prime}\right) \bmod 2, t_{z}^{\prime}\right)
$$

where $t^{\prime}=\left(t_{x}^{\prime}, t_{y}^{\prime}, t_{z}^{\prime}\right)$ is the thread in local space. The map $c()$ assumes that blocks have even dimensions, which in practice is not a limitation since it is typically a requirement for efficient thread-blocks. Figure 3 illustrates the computation in shared memory being organized in four steps. The first step, (1) block to $S M$, consists of copying the block of spins from global to
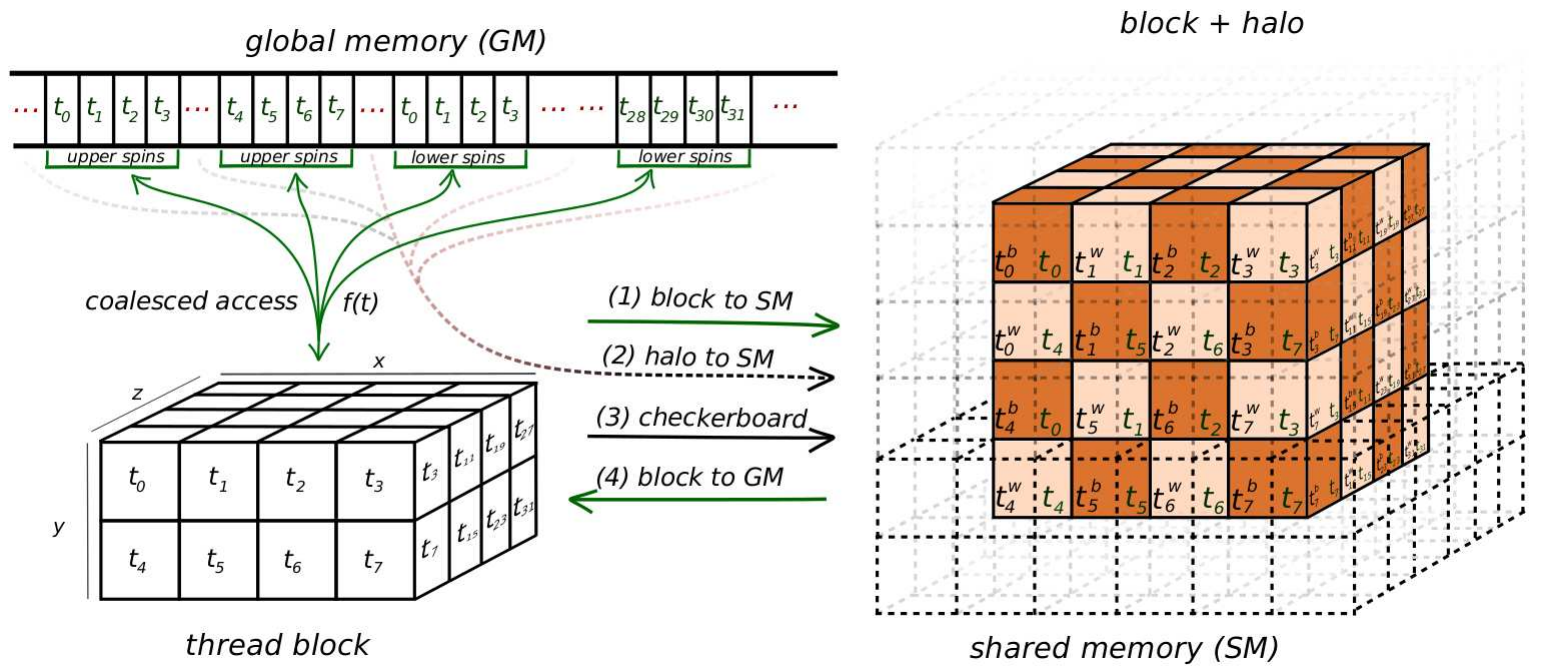

Figure 3: Threads are mapped differently depending if they are copying from/to global memory or doing the Metropolis-Hastings computation. A warp of four threads was assumed for illustration purposes.

shared memory using the coalesced map $f()$ which is shown as green color arrows from threads to global memory. The corresponding copy locations in shared memory are given by the green thread indices at the bottom right corner of each spin in the SM block. The second step, (2) halo to $S M$, consists of copying a 3D halo of spins from global to shared memory that surrounds the block in SM. These spins are located in between the rows of spins read by step one. The $X Y$ and $X Z$ halo sides can be copied with just one coalesced access, but the $Y Z$ halo spins are copied less efficiently since a full memory access is employed by just one thread of the boundary warp. For this reason it is better to favor the length of the $X$ dimension of the shared memory block and make an halo composed mostly by $X Y X Z$ halo spins. The third step, (3) checkerboard, consists of doing the actual simulation of the spins using the checkerboard map. The corresponding location for each thread in SM is shown in black 
color at the bottom left corner of each spin, where $t_{i}^{b}$ and $t_{i}^{w}$ refers to the $i$-th thread doing the black and white simulations steps, respectively. The double checkerboard approach has a special feature which is the possibility of doing several checkerboard updates during step (3), taking advantage of the shared memory bandwidth. This idea is known as the multi-hit update technique and was proposed by Weigel $[29,28]$. It is important to clarify that even if just $K=1$ multi-hit updates are used, the double-checkerboard is still convenient in terms of performance since each spin (except for the boundary ones) is accessed at least five times (four times by its neighbors and one by itself) and written if it is flipped. Doing all these memory accesses in shared memory is faster than doing it in global memory, specially if the copy from/to global memory can be done with a coalesced access as well. The last step, (4) block to GM, copies the block back to global memory (GM) in a coalesced way as in step (1). The halo is not copied back to GM since it is read-only.

\subsubsection{Optimal Min-Halo Thread-block}

The halo spins, which are shown as dashed cubes in Figure 3, introduce an additional cost in memory accesses which is unwanted, since these spins are not simulated, but instead just accessed by the spins that are on the boundary of the block. For this reason, one would want to minimize the number of halo spins in the shared memory block while keeping a large interior volume. Considering that actual GPU architectures have a constant warp of threads of $w=32$, we found convenient to fix $B_{x}^{s}=w$ on the shared memory block, since it will also produce an halo composed mainly by $X Y$ and $X Z$ spins which will favor performance. Finding the minimum halo (min-halo) is equivalent to solve the optimization problem of minimizing the surface of a closed box with dimensions $w \times y \times z$ and volume $\left|B^{s}\right|$. The objective function to minimize is the surface of the block

$$
S=2(w y+z y+w z)
$$

subject to $\left|B^{s}\right|=w y z$, from where we can rewrite $S$ just in $y$ given that $z=\left|B^{s}\right| / w y$. Setting the derivative of $S$ to zero leads to

$$
\frac{\partial S}{\partial y}=2\left(w-\left|B^{s}\right| / y^{2}\right)=0
$$

where the solution is $y=\sqrt{\left|B^{s}\right| / w}$, which is the same for $z$. Considering that the number of spins inside a checkerboard is double the number of threads and that in the Kepler GPU architecture the maximum number of threads in a block is $\left|B^{t}\right|=1024$, we can establish that the shared memory block can have up to $\left|B^{s}\right|=2048$ spins being processed in parallel without having to increase the work per thread. With this, we finally have that $y=z=8$, the optimal shared memory block is $B^{s}=(32,8,8)$ thus the optimal thread-block is

$$
B_{o p t}^{t}=\left(B_{x}^{s}, B_{y}^{s} / 2, B_{z}^{s}\right)=(32,4,8)
$$

\subsubsection{Analysis of Bank Conflicts}

It is worth noticing that although the checkerboard map does not place threads in a typical pattern for accessing shared memory and the halo spins introduce a +2 increment to each dimension of the shared memory block, the computation does not compromise the shared memory bandwidth efficiency as threads still access the banks $b_{0}, b_{1}, \ldots, b_{w-1}$ without producing any bank conflicts. Figure 4 shows how a single warp of 32 threads accesses the 


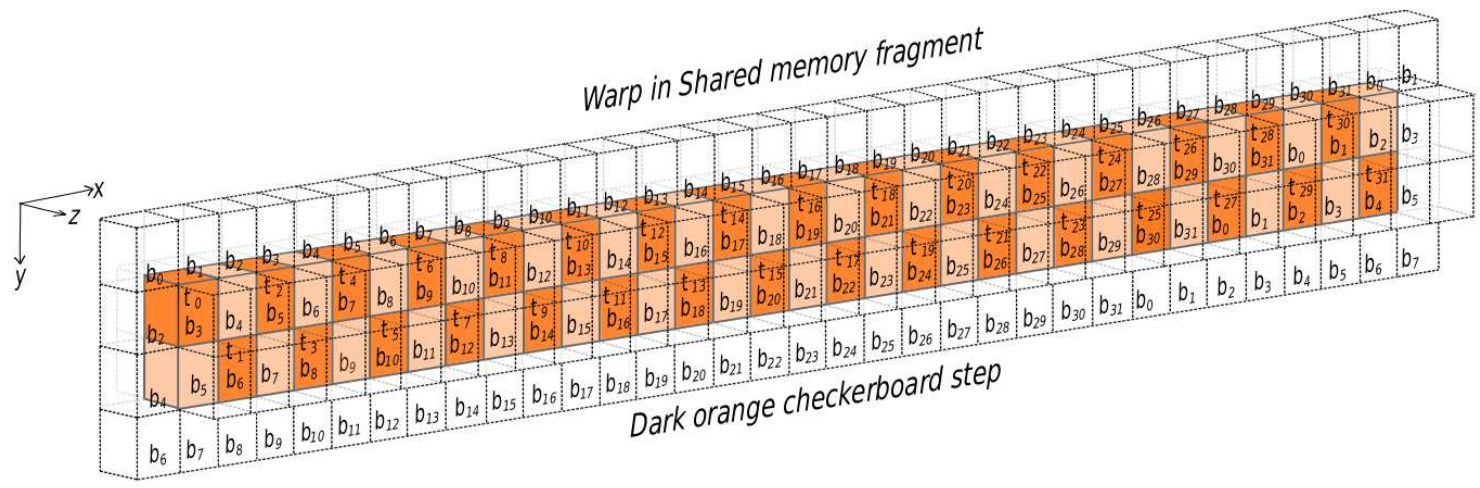

Figure 4: The checkerboard map does not produce any bank conflicts when accessing a 3D block + halo in shared memory.

bank slots when processing the dark orange spins. From the Figure one can observe that threads in a warp map onto unique bank slots when accessing their corresponding center and neighbor spins, regardless of the fact that odd and even threads map to the first and second row of the corresponding $X Y$ plane, respectively. For other $X Y$ planes at different $Z$ the pattern is kept, with the only difference that there will be an offset on the indices of the bank slots, but it does not compromise the efficiency of memory accesses, since they are free of bank conflicts too. The analysis is analogous when the threads switch to the light orange spins.

\subsubsection{Arithmetic Encoding of Spin Flips}

Thread branching ${ }^{1}$ is a run-time phenomena that can penalize the performance of massively parallel processors such as the GPU. It occurs when threads within a warp take different paths of execution as a result of a conditional instruction evaluated differently throughout the warp. As a result, each generated branch is executed sequentially for the given warp. To prevent thread branching, the kernel code has to be free of potential thread branching conditionals. For the case of the Metropolis-Hastings algorithm, potential thread branching arises at the conditional for the flipping part where a spin $s_{i}$ can change to its contrary state $s_{i}^{\prime}$ with probability

$$
P\left(s_{i} \Rightarrow s_{i}^{\prime}\right)=\left\{\begin{aligned}
1 & , \Delta \mathcal{H}\left(s_{i}\right) \leq 0 \\
\mathrm{e}^{\frac{-\Delta \mathcal{H}\left(s_{i}\right)}{\kappa T}} & , \Delta \mathcal{H}\left(s_{i}\right)>0
\end{aligned}\right.
$$

where $\Delta \mathcal{H}\left(s_{i}\right)=\mathcal{H}\left(s_{i}^{\prime}\right)-\mathcal{H}\left(s_{i}\right)$ is the difference of the local Hamiltonians of $s_{i}^{\prime}$ and $s_{i}$, respectively. For a given spin $s_{i}$, the local Hamiltonian

$$
\mathcal{H}\left(s_{i}\right)=-J \sum_{\langle i, j\rangle} s_{i} s_{j}+h \cdot h_{i} s_{i}
$$

computes the local energetic contribution of spin $s_{i}$ with its nearest neighbors $\langle i, j$,$\rangle and its$ corresponding magnetic field value $h_{i}= \pm 1$ with strength $h$. The conditional involved in

\footnotetext{
${ }^{1}$ Technically speaking, whole warps taking different execution paths is also considered thread branching, but we do not refer to this case as it does not produce any performance degradation.
} 
expression (10) can be avoided in the kernel by encoding the flip into the following arithmetic form causing no branching at all:

$$
s_{i} \leftarrow s_{i}\left(1-2\left[\omega\left(\Delta \mathcal{H}\left(s_{i}\right)\right) \vee \omega\left(\operatorname{rand}()-\mathrm{e}^{\frac{-\Delta \mathcal{H}\left(s_{i}\right)}{\beta}}\right)\right]\right)
$$

Function $\omega(x)$ returns the sign bit of $x$, i.e, 0 for $x \geq 0$ and 1 for $x<0$, Operator $\vee$ is the bitwise OR and $\operatorname{rand}()$ returns a random float using a uniform distribution in the range $[0,1]$. Function $\omega(x)$ can be implemented as

$$
\omega(x)=x \oplus(1 \ll d)
$$

where $\oplus$ is the binary XOR operator, $1 \ll d$ is the bit-shift of value $1, d$ positions to the left, to match the position of the sign bit in the IEEE 754 floating point standard [39]. Alternatively, one can use the signbit $(x)$ function that is already implemented in CUDA.

\subsection{Pseudo Random Number Generator}

The present work is not tied to any particular pseudo random number generator (PRNG) and it can be implemented using a simple linear congruential generator (LCG). A LCG indeed helps in terms of performance, but in terms of quality it is not as good as other PRNGs available. The PRNG considered for this work is a GPU implementation of the recent permuted congruential generator (PCG) proposed by M. O'Neill [40], which is a fast PRNG that combines permutation functions and a base LCG. The idea of the PCG is to pass the output of a fast LCG through a hash function to enhance the quality of the output. A $2 b$-bit PCG generates $b$-bit outputs with a period of $2^{2 b}$ and supports up to $2^{b-1}$ streams. If $b=32$ bits, we have 64 -bit PCG states with a period of $2^{64}$ and $2^{31}$ possible streams. The $b=32$ PCG can be implemented efficiently on the GPU as each PCG state becomes a 16-byte structure that can be accessed in a fully coalesced way. The CURAND XORWOW [41] was considered too since it is a fast PRNG, but it was later discarded because it used 48-bit states, giving slow performance due to the non-coalesced memory accesses.

In the next sub-section we specify how the simulation of many replicas are organized in a multi-GPU scheme.

\subsection{Replica Level Parallelism}

Replica level parallelism is achieved by the combination of concurrent kernel execution from modern GPUs and coarse parallelism from the multi-GPU computing. In modern GPU architectures, one can launch multiple kernels in the same GPU and let the driver scheduler handle the physical resources to execute these kernels concurrently for that GPU. Starting from the Kepler GPU architecture, it is possible to launch up to 32 kernels concurrently on a single GPU. The idea is to divide the $R$ replicas into $k$ available GPUs and simulate $\rho=\lceil R / k\rceil$

replicas concurrently on each GPU. It is possible that $\rho>32$, but it is not a problem since the GPU can handle the exceeding kernels automatically with an internal execution queue. With this approach, the number of replicas becomes

$$
R^{\prime}=D \cdot \rho \leq R+D
$$

where $D$ is the number of GPUs used in the multi-GPU computation. One assumes that $D \leq R$. The multi-GPU configuration presents a distributed memory scenario that is handled 
with a global indexing of the local memory regions. For each GPU, there is a region of memory allocated for the $\rho$ replicas. For any GPU $D_{i}$, the global index for its left-most replica is $D_{i}^{L}=\rho \cdot i$ while the global index for its right-most replica is $D_{i}^{R}=\rho \cdot i+\rho-1=\rho(i+1)-1$.

Replica exchanges that occur in the same GPU are efficient since the swap can just be an exchange of pointers. However in the limit cases at $D_{i}^{L}$ and $D_{i}^{R}$, the task is not local to a single GPU anymore as the exchange process would need to access replicas $D_{i-1}^{R}$ and $D_{i+1}^{L}$, both which reside in different GPUs. Because of this special case, the pointer approach is not a robust implementation technique for a multi-GPU approach, neither explicit spin exchanges since they would require several memory transfers from one GPU to another. A typical optimization technique done for PT is to swap temperatures instead of replicas [42], which is totally equivalent for the result of the simulation. One just needs to keep track of which replica has a given temperature and provide a mechanism to access neighbors. Two index arrays are used for this purpose, trs and $r t s$, for accessing temperatures replica-sorted and replicas temperature-sorted, respectively. Figure 5 illustrates an example how both index arrays serve in the process of accessing the corresponding temperature of a given replica and vice versa, for a multi-GPU system of four GPUs $\left\{D_{0}, D_{1}, D_{2}, D_{3}\right\}$.

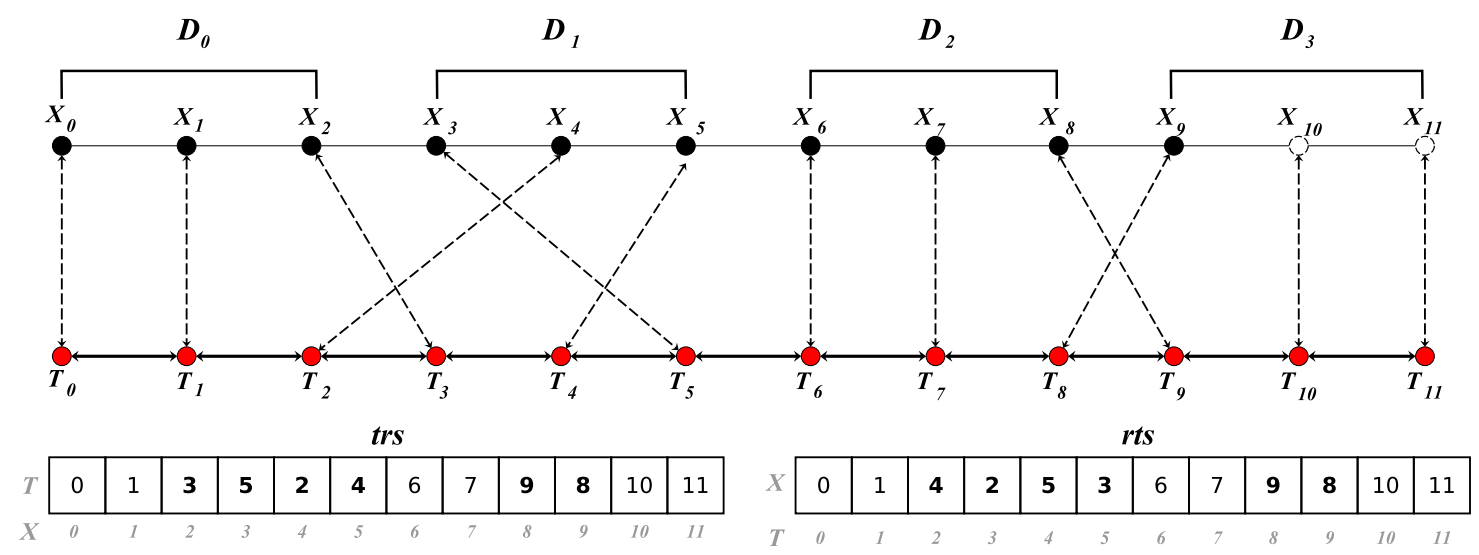

Figure 5: An ensemble of replicas being simulated in a multi-GPU system of four GPUs, with some temperatures already swapped. The trs index array provides the corresponding temperature for a given replica, while $r$ s provides the inverse.

The example illustrates an ensemble of replicas during a simulation (10 replicas padded to 12 replicas to match the multiplicity of 3 replicas per GPU in this case), with some temperatures already swapped. At any stage of the simulation, trs will return the index of the corresponding temperature for any replica index, and rts will return the index of the corresponding replica for any temperature index. Tasks such as the Metropolis-Hastings simulation as well as measuring the physical observables, require a replica-based order organization in order to match the memory spaces of each GPU. For those tasks the corresponding temperature of each replica is obtained with the trs array. On the other hand, storing the physical observables requires a temperature order for an eventual plotting; in this case the corresponding replicas are accessed with the rts array.

The temperature swap approach benefits distributed memory systems such as a multiGPU system and clusters of nodes, since for each exchange only two floating point values (i.e., $2 \times 32 b i t s$ ) need to be transferred to accomplish the swap. Since swapping results in little overhead compared to the simulation time, we opted to implement the exchange of 
temperatures as a sequential CPU function. It is important to clarify however that the energy measurements involved in the exchange phase are done in parallel using GPU reductions based on the shuffle approach [43].

\section{Adaptive Temperatures}

The exchange Monte Carlo algorithm has been redesigned as a two-phase process, that begins with an adaptation phase; where the temperature set is built, and is followed by a simulation phase; which is the original part of the algorithm where physical quantities are measured and results extracted. This Section focuses on the relevant aspects of the adaptation phase.

At the low-temperature regime and near the critical point $T_{c}$, the temperature gaps between two neighbor replicas cannot be as large as in the high temperature regime, otherwise the simulation suffers from low exchange rate bottlenecks that compromise the physical results. Indeed one can decide to handle the problem using a dense set of temperatures where the gaps meet the required distance, but such strategy would require an unnecessary amount of computation. Furthermore, too much exchange rate could produce a negative effect on the physical results as replicas may do several unwanted movements.

The study of the exchange rates across the temperature set is an aspect of great interest, since the quality of the simulation depends in great part on how are the temperature points distributed. Earlier works have found that an exchange rate of $20 \%$ or $23 \%$ is good enough for the parallel tempering method to work as expected [44, 45]. In our case, after several empirical tests with different parameters, we found that the range $20 \% \sim 40 \%$ was effective for simulating the 3D RFIM with accurate results. Once the adequate exchange rates are known, the remaining question is how one can build a temperature set that provides the required exchange rate. We employ an adaptive strategy for this, that integrates to the multi-GPU design. The adaptive strategy is based on the feedback principle used by Katsgraver et. al. [37] and Bittner et. al. [38], but instead of adapting the total number of temperatures from the beginning, we propose a simple approach based on the divide and conquer strategy, that inserts temperatures as they are required during the adaptation phase. This approach has the advantage of requiring less work during the trial simulations, since the process works with a subset of the total number of replicas. The idea of the approach is to start with a small number of $R^{*}<R$ replicas arbitrarily distributed from $T_{\text {low }}$ to $T_{\text {high }}$. The adaptive method performs an arbitrary number of small trial simulations to measure the average exchange rate at each temperature gap. After each trial simulation, a histogram of exchange rates is obtained and put into a min-heap. For the $a$ intervals with lowest exchange rate (with $a$ chosen arbitrarily), new replicas are introduced at their mid-points. Figure 6 illustrates the process when additional replicas are required near $T_{c}$, for an ensemble of $R^{*}=8$ replicas, using three trial runs and $a=4$.

The number of replicas handled at the $i$-th trial run is given by

$$
R_{i}^{*}=R_{i-1}^{*}+a
$$

and satisfies $R_{i}^{*} \leq R$ for all trial steps. Although the adaptive method used in this work is simple, implementing it as a multi-GPU computation introduces some additional considerations that are worth mentioning. Given that the number of trial runs and the number of mid-point insertions are given a priori, the memory pool can be allocated before hand and 


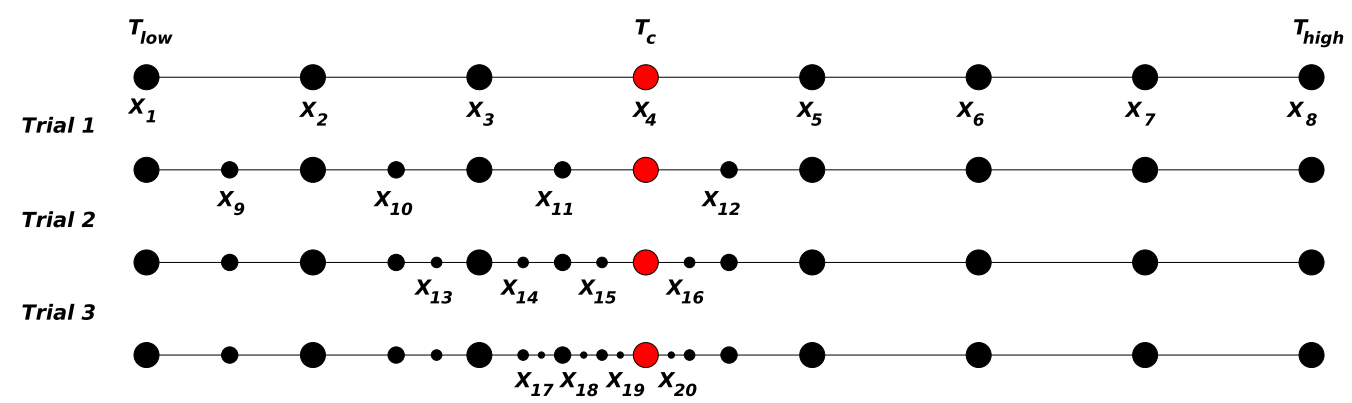

Figure 6: The adaptive temperature strategy, employing three trial runs with mid-point insertions on the lowest four exchange rates.

distributed among the available GPUs. After each trial run, the $i$-th new replica is assigned to the $j$-th GPU with $j=i \bmod k$, where $k$ is the number of GPUs. The order in which new replicas are assigned to each GPU actually does not affect the performance neither the result of the simulation. Figure 7 illustrates the memory layout for the same example shown in Figure 6.

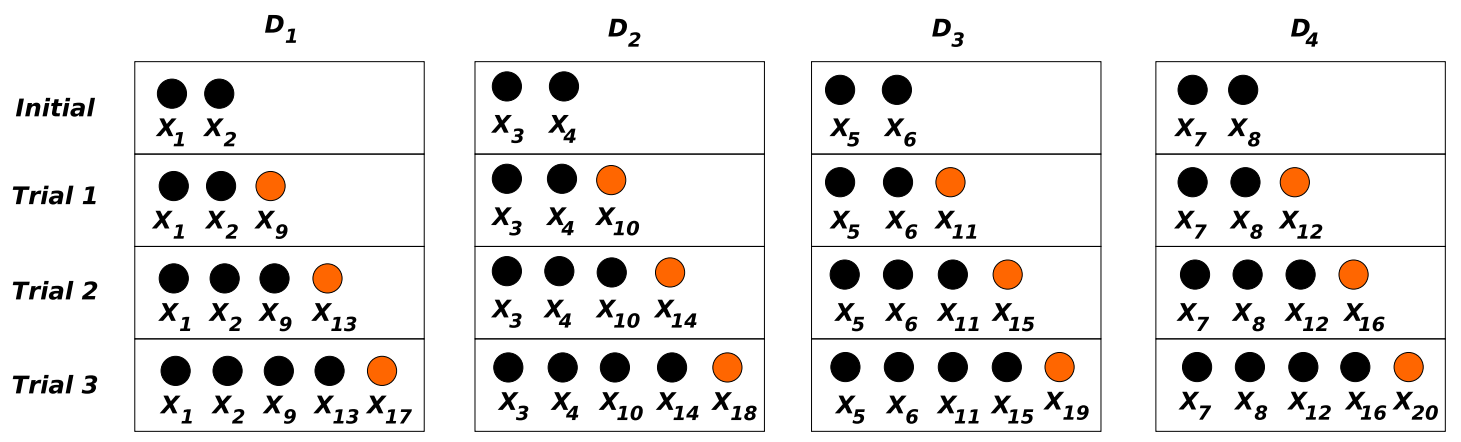

Figure 7: Replica insertion in a system of four GPUs; $\left\{D_{1}, D_{2}, D_{3}, D_{4}\right\}$. Incoming replicas (in orange color) can be inserted in a balanced way, no matter if they belong to a low or high temperature regime. to maintain the parallel multi-GPU efficiency.

At each trial run, new replicas (in orange color) are inserted into the different memory spaces of each GPU. The goal is to keep the computation balanced for each GPU so that parallel multi-GPU efficiency is not compromised. The corresponding temperature mappings are achieved by the rts and trs index arrays explained in the previous Section. Once the adaptive process has finished, the mapping between replicas and temperatures can be reset to a simple linear correspondence before the simulation begins, i.e., $\operatorname{rts}[i]=\operatorname{trs}[i]=i$.

\section{Technical Details About the Implementation}

The implementation is named trueke for exchange in Spanish, which is trueque, and was programmed with CUDA 7.0 [43] and OpenMP 4.0 [46]. The multi-GPU functionality is managed by OpenMP threads, where one thread is in charge of one GPU. The set of replicas and tables of physical values are properly divided according to the number of threads and memory spaces ensuring that no false sharing occurs between the OpenMP threads. 
The source code of the implementation is maintained at git-hub and is available to the community at https://github.com/crinavar/trueke. The program has been tested on Geforce Nvidia GTX 765M, GTX 760, Tesla K20 and Tesla K40c GPUs. There is also a multi-node MPI version available, named mpitrueke, that can run across several computers and is available at https://github.com/crinavar/mpitrueke. The mpitrueke implementation uses a third parallelization level, where each MPI process averages over an arbitrary number of disorder realizations chosen as parameter, followed by a parallel MPI reduction of these averages to get the final average of all realizations. The Linux distributions tested were Arch Linux 64-bit and Fedora Server 21. The MPI implementation used was open-mpi-1.10.1.

\section{Performance results}

In this section we present the performance results of trueke and compare them against other GPU and CPU implementations.

\subsection{Benchmark Plan}

The performance metrics chosen measure the parallel performance of trueke and are defined as average values taken from $N$ repetitions (Note, symbol $Q$ was chosen as the running time to avoid confusion with the temperature symbol, $T$ ):

1. Spin-level Performance is the average time of a spin flip, defined as

$$
Q_{s}=\frac{1}{w N L^{3}} \sum_{i=1}^{N} Q_{w}
$$

where $Q_{w}$ corresponds to the time spent doing $w$ Metropolis-Hastings sweeps.

2. Normalized Replica-level Performance is the average time of a parallel tempering realization per replica:

$$
Q_{r}=\frac{1}{N R} \sum_{i=1}^{N} Q_{x}
$$

where $R$ is the number of replicas and $Q_{x}$ is the time spent doing an arbitrary number $x$ of parallel tempering steps.

3. Multi-GPU Performance Scaling is obtained by measuring, based on the single GPU and multi-GPU running times $Q_{1}, Q_{g}$, the fixed-size speedup $S_{G P U}$ and efficiency $E_{G P U}$

$$
\begin{aligned}
S_{G P U} & =Q_{1} / Q_{g} \\
E_{G P U} & =S_{G P U} / g
\end{aligned}
$$

at different problem sizes when using two $(g=2)$ GPUs.

4. Adaptive Temperatures Performance is the average time of an adapted parallel tempering realization:

$$
Q_{\text {adapt }}=\frac{1}{N} \sum_{i=1}^{N}\left(Q_{k}+Q_{x}\right)
$$

where $Q_{k}$ is the time for doing $k$ trials. 
A total of $N=10$ repetitions were used and the standard error obtained in all performance measurements was in the order of $1 \%$. In addition, we also show how the performance increases when using faster GPUs as well as when using more multi-hit updates. The workstation used for all benchmarks (including the comparison implementations) is equipped with two 8-core Intel Xeon CPU E5-2640-V3 (Haswell), 128GB of RAM and two Nvidia Tesla K40c.

\subsection{Spin and Replica level Performance Results}

Figure 8 presents four performance results; (1) the average spin-level performance with $K=1$ multi-hit updates, (2) spin-level performance with $K \in$ [2..100] multi-hit updates, (3) single GPU performance scaling and (4) normalized replica-level performance.

The spin and replica level performance results are compared, for reference, against a cachealigned CPU implementation of the 3D Random Field Ising model running both sequential and multi-core, denoted $C P U_{r, s}$ where $r$ specifies the number of replica-level parallel threads and $s$ the spin level parallel threads. Additionally, we include the Spin-level performance of Weigel's GPU implementation for the 2D Ising model running on the same system to observe how different is the performance of the double checkerboard approach when moving from 2D Ising to 3D RFIM. The lattice sizes of Weigel's implementation have been adapted to the form $L=\sqrt[3]{L_{2 d}^{2}}$, where $L_{2 d}$ is the original linear size for the two-dimensional case. This way, the performance of Weigel's original work can be plotted and compared against trueke's performance in the number of total spins. As an example, a Weigel's $2 \mathrm{D}$ size of $L_{2 d}=512$ corresponds to a $3 \mathrm{D}$ size of $L=64$ in the plot.

On the top-left panel, $L$ corresponds to the linear size of a $3 \mathrm{D}$ system, i.e, $L^{3}$ spins are being simulated. One can observe a notorious difference between the GPU and CPU implementations, of at least an order of magnitude with respect to the multi-core implementation running on 16 cores, and at least two orders of magnitude compared to the sequential $\mathrm{CPU}$ one. For the GPU implementations, trueke is close to the performance of Weigel's highly optimized GPU Metropolis implementation for the 2D Ising model. The performance gap between Weigel's 2D implementation and trueke comes in part from the cost of flipping spins in $3 \mathrm{D}$ space using a random field and also from the chosen PRNG which is more expensive than one based only on a LCG.

In the top-right panel one can observe that the performance gap between trueke and Weigel's implementation is significantly reduced when employing at least $K=10$ multi-hit updates instead of one, and the behavior is more notorious for larger lattice sizes as $L=256$. The lattice sizes $L_{2 d}=512$ and $L_{2 d}=4096$ match with $L=64$ and $L=256$ in the number of total spins, respectively.

The bottom-left panel shows the spin-level performance scaling when increasing the number of cores per GPU, while Table 1 presents the main features of each GPU. From the plot

Table 1: Specifications of the GPUs used.

\begin{tabular}{|c|c|c|c|l|}
\hline GPU & CUDA cores & Frequency $(\mathrm{MHz})$ & Interface Width & Memory Bandwidth \\
\hline GTX 765M & 768 & 850 & $128-\mathrm{bit}$ & $64 \mathrm{~GB} / \mathrm{sec}$ \\
GTX 760 & 1152 & 980 & $256-\mathrm{bit}$ & $192.2 \mathrm{~GB} / \mathrm{sec}$ \\
Tesla K20 & 2496 & 706 & $320-\mathrm{bit}$ & $208 \mathrm{~GB} / \mathrm{sec}$ \\
Tesla K40 & 2880 & 745 & $384-\mathrm{bit}$ & $288 \mathrm{~GB} / \mathrm{sec}$
\end{tabular}



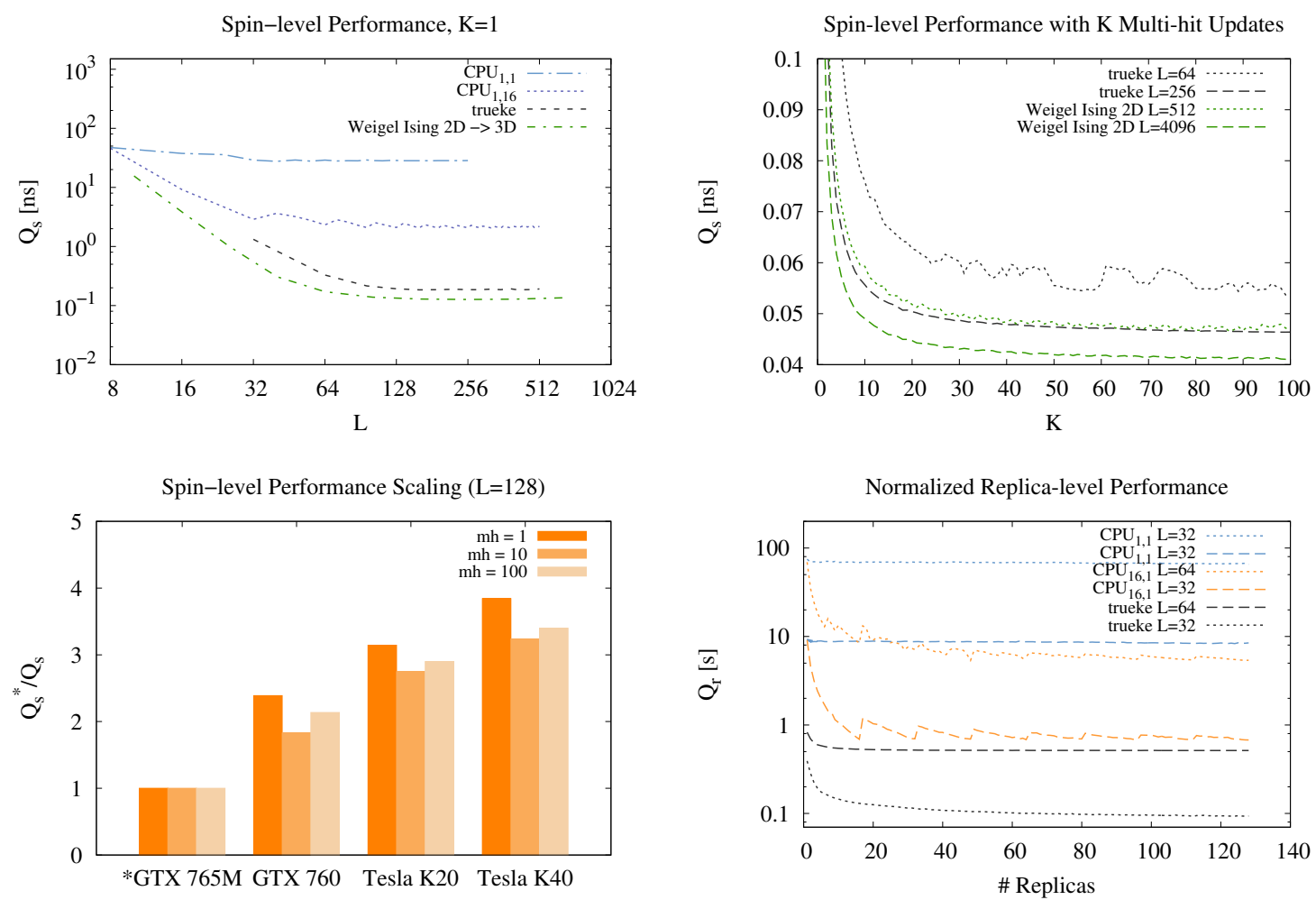

Figure 8: Top-left: Comparison of spin-level performance of different implementations. Top-right: spin-level performance as more multi-hit updates are employed. Bottom-left: performance scaling with more cores per GPU. Bottom-right: normalized replica-level performance.

and table one can note that the scaling is proportional to the increment in the number of cores and memory bandwidth. It is highly important that GPUs with higher core count also have higher memory bandwidth, since the Metropolis-Hastings algorithm is considered a memory bound problem.

The bottom-right panel presents the normalized replica-level performance for the sequential CPU, multi-core CPU and trueke implementations. The results show that in the case of trueke the GPU's concurrent kernel execution favors small sized lattices, such as $L=32$, leading to an improved performance per replica. For larger lattice sizes the effect is expected to be weaker, as in the case of $L=64$, since each time the simulation of a single replica will produce a higher GPU occupancy. Although the GPU performance is significantly superior than both CPU implementations, one can note that the multi-core implementation improves the normalized replica-level performance by an order of magnitude with respect to the sequential CPU one, and does not degrade even when the exchange part of the algorithm is done sequentially.

\subsection{Multi-GPU Scaling}

Figure 9 presents the speedup and efficiency of trueke using two Tesla K40 GPUs.

The goal of measuring multi-GPU performance is to study how the performance scales with multiple GPUs considering that there is an execution overhead coming from the sequential cost of the exchange part, and the cost of OpenMP threads, one per GPU. There are also parallel 

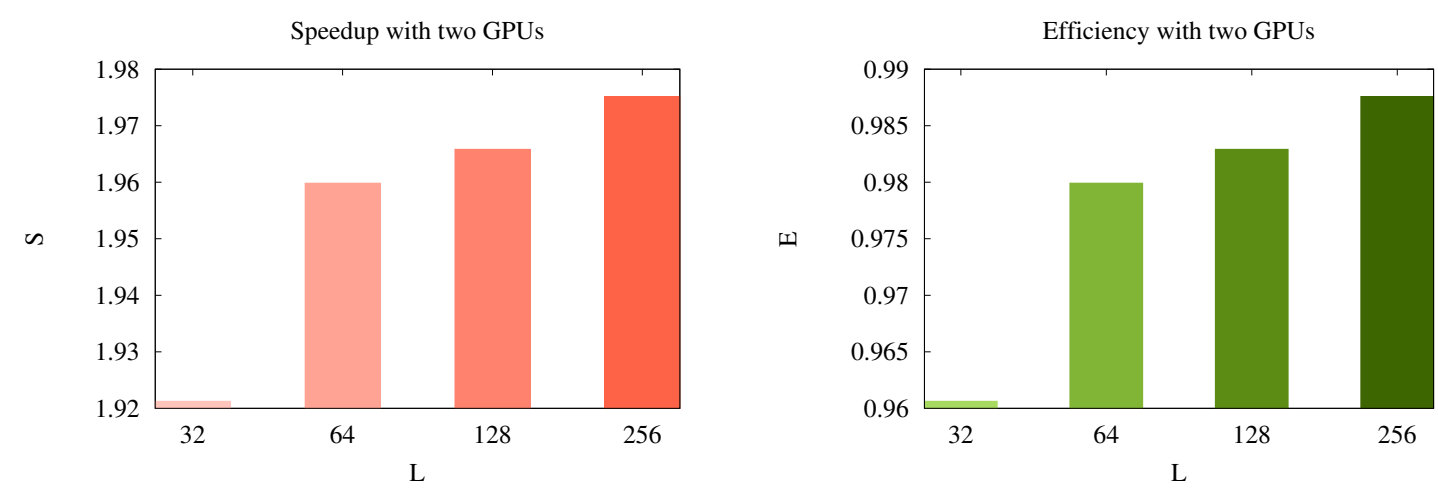

Figure 9: Multi-GPU speedup and efficiency for different lattice sizes.

reductions involved in order to measure the energy and magnetization of each replica. From the results, one can observe that replica-level speedup approaches $S_{G P U}=2$ as $L$ increases, which is reflected as an efficiency close to $E_{G P U}=1$. The results indicate that replica-level parallelism does not degrade when increasing $L$. This behavior can be explained in part by the fact that the work of the exchange phase grows at least as $W_{e x}=\Omega(R)+\Omega\left(R L^{3}\right)$ where the $\Omega(R)$ term is the sequential work for exchanging the $R$ replicas, while the $\Omega\left(R L^{3}\right)$ term is the parallel work for computing the energy at each replica, which is done with a parallel GPU reduction, in $O\left(\log \left(L^{3}\right)\right)$ time for each replica. It is clear that the amount of parallel work grows faster than the sequential work, therefore the parallel efficiency of the whole method should not degrade as long as both the number of GPUs and $L$ increment together.

\subsection{Performance of The Adaptive Temperatures Technique}

Table 2 presents the running times of the adaptive temperatures technique compared to dense and spare homogeneous techniques for $L=32,64$. The simulation parameters used for all simulations were 100 disorder realizations, each one with 2000 parallel tempering steps and 10 Metropolis sweeps. For $L=32$, the adaptation phase used 10 trial runs with 2 insertions at each trial. For $L=64$, the adaptation used 32 trial runs, with 3 insertions at each trial. The trial runs also use 2000 parallel tempering steps with 10 Metropolis sweeps.

Table 2: Executions times, in seconds, for adaptive and homogeneous approaches.

\begin{tabular}{c|c|c|c|c|c}
\hline $\mathrm{L}$ & sparse-sim & dense-sim & adapt-trials & adapt-sim & adapt (trials + sim) \\
\hline 32 & 670.25 & 1417.04 & 41.84 & 1167 & 1209.49 \\
64 & 10386.75 & 26697.17 & 1689.46 & 19994.70 & 21684.15
\end{tabular}

From the results of Table 2, one can observe from the point of view of performance that a full adaptive simulation, including its adaptation time, is more convenient than a dense simulation with no adaptation. The sparse technique is the fastest one because it simply uses less replicas, but in the next Section we show that it cannot exchange at the low temperature regime. 


\section{Exchange Rates with Adaptive Temperatures}

Results on the exchange rate of the adaptive strategy are presented in Figure 10 (left) showing the evolution of the maximum (Max), average and minimum (Min) exchange rates through the trial runs. The right side of Figure 10 presents a comparison of the exchange rates between dense uniform, adaptive and a sparse uniform sets. The simulation parameters were the same as the ones used for the performance results showed on Table 2.
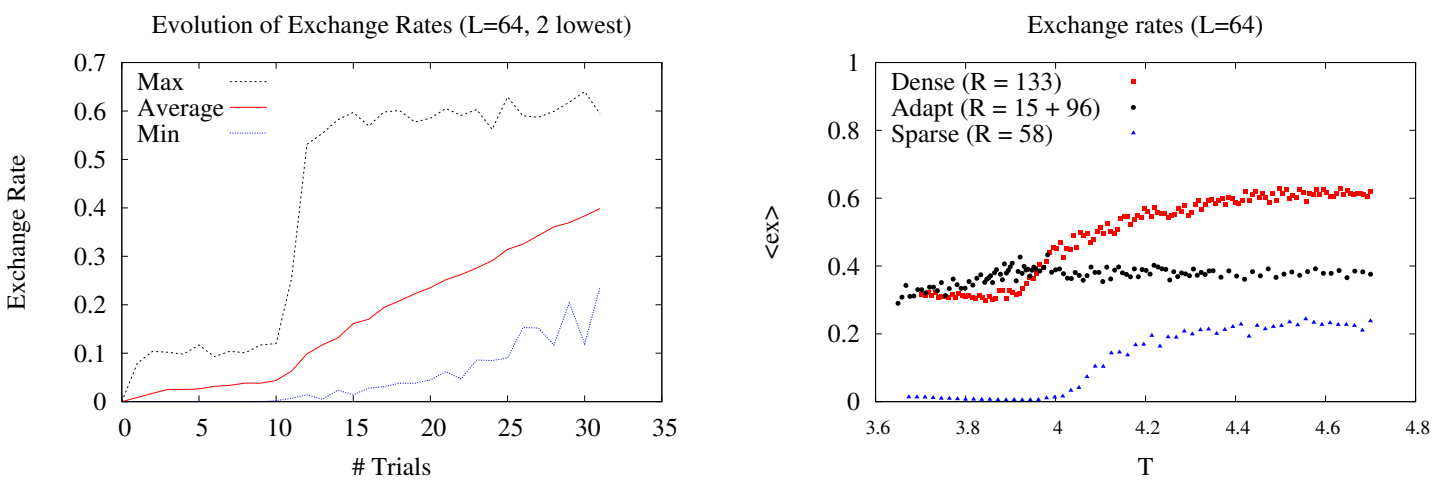

Figure 10: On the left, the evolution of maximum (Max) average and minimum (Min) exchange rates through the trial runs. On the right, the exchange rates for the whole temperature range using dense, adaptive and sparse approaches.

From the left plot we observe that as more replicas are added, the average and minimum exchange rates increase and tend to get closer, while the maximum exchange rate stabilizes from trial number 10. For the right plot, it is clearly shown that the dense approach, although it has a uniform distribution of temperatures, does not generate a uniform exchange rate value across the temperature range. For the sparse approach the scenario is even worse because at the low temperature regime there is almost no exchange. The adaptive method generates an exchange rate that is close to uniform for the entire range. The numbers on the labels indicate the number of replicas used, where one can see that the adaptive method uses less replicas than the dense uniform.

\section{Physical Results}

Trueke was used to obtain physical results for sizes up to $L=64$. The involved simulations used CUDA's XORWOW PRNG instead of the PCG PRNG, because back then PCG was still not considered. The physical quantities are average values of many disorder realizations. The physical observables obtained from one realization are average values of a full parallel tempering process. For the physical quantities, the following definitions were used:

The magnetization $M$ is defined as

$$
M=\left|\frac{1}{V} \sum_{i=1}^{L^{3}} s_{i}\right|
$$

Where $V=L^{3}$ is the lattice volume. The specific heat is

$$
C=\frac{L^{3}}{T^{2}}\left(\left\langle E^{2}\right\rangle-\langle E\rangle^{2}\right)
$$


where $\left\langle E^{2}\right\rangle$ is the average squared per-site energy and $\langle E\rangle^{2}$ the squared average per-site energy. The susceptibility is

$$
\chi=\frac{L^{3}}{T}\left(\left\langle M^{2}\right\rangle-\langle M\rangle^{2}\right)
$$

where $\left\langle M^{2}\right\rangle$ is the average squared magnetization and $\langle M\rangle^{2}$ the squared average magnetization. The Binder factor is an average that samples at the disorder realization level and it is defined as:

$$
g=\frac{1}{2}\left(3-\frac{\left[\left\langle M^{4}\right\rangle\right]}{\left[\left\langle M^{2}\right\rangle\right]^{2}}\right)
$$

where $\left[\left\langle M^{4}\right\rangle\right]$ is the disorder realization average of the fourth-power magnetization and $\left[\left\langle M^{2}\right\rangle\right]^{2}$ is the squared disorder realization average of the squared energy. The definition of correlation length corresponds to the one used by Salas and Sokal [47] (eq. 2.8 of their work),

$$
\xi=\frac{1}{2 \sin (\pi / L)}\left(\frac{\left\langle M^{2}\right\rangle}{\langle F\rangle}-1\right)^{1 / 2}
$$

with $\langle F\rangle$ being an average denominator for one disorder realization, defined as

$$
\begin{aligned}
F & =\frac{1}{3 L^{3}}\left(F_{1}+F_{2}\right) \\
F_{1} & =\left(\sum_{i=1}^{L^{3}} s_{i} \cos \left(K \cdot i_{x}\right)\right)^{2}+\left(\sum_{i=1}^{L^{3}} s_{i} \cos \left(K \cdot i_{y}\right)\right)^{2}+\left(\sum_{i=1}^{L^{3}} s_{i} \cos \left(K \cdot i_{z}\right)\right)^{2} \\
F_{2} & =\left(\sum_{i=1}^{L^{3}} s_{i} \sin \left(K \cdot i_{x}\right)\right)^{2}+\left(\sum_{i=1}^{L^{3}} s_{i} \sin \left(K \cdot i_{y}\right)\right)^{2}+\left(\sum_{i=1}^{L^{3}} s_{i} \sin \left(K \cdot i_{z}\right)\right)^{2}
\end{aligned}
$$

where $K=2 \pi / L$ and $\left\{i_{x}, i_{y}, i_{z}\right\}$ correspond to the spatial coordinates of a given spin $s_{i}$ in the lattice.

The relevant parameters are listed and defined in Table 3.

Table 3: Simulation parameters.

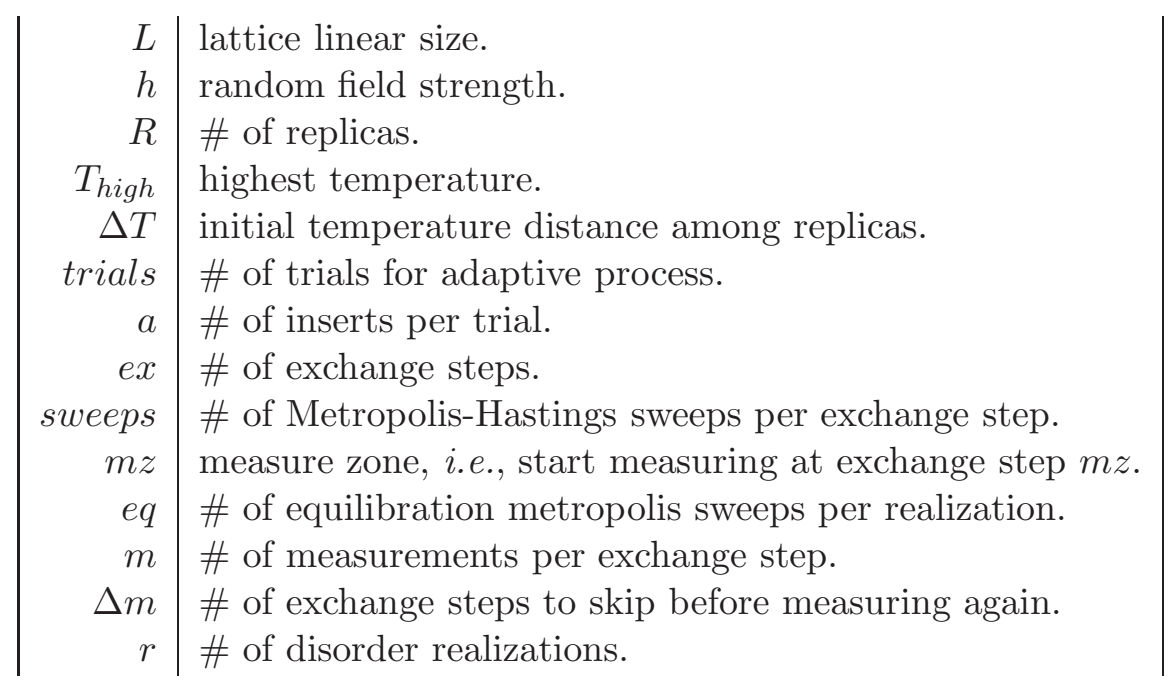


At each exchange, we considered from 1 to 10 Metropolis-Hastings sweeps (sweeps) since it is a range that has worked in the past [27]. We used only $K=1$ multi-hit updates for all simulations. A value of $K=100$ multi-hit updates is very unlikely to help the simulation, however smaller values in the range $K=1 . .10$ can be of great help even for disordered systems [48], improving performance without sacrificing quality. Studying the 3D RFIM with a moderate number of multi-hit updates is an aspect yet to be studied, and trueke can work with this feature. For the exchange rates, we used a lower bound of approximately $20 \%$ based on the earlier works mentioned in an earlier Section [44, 45] and an upper bound of $45 \%$ which was found experimentally. Through experimentation, we found different sets of parameters for different values of $L$. The ones presented in Table 4 gave us accurate physical results.

Table 4: Chosen parameters for different $L$.

\begin{tabular}{|c|c|c|c|c|c|}
\hline $\mathrm{h}$ & $\mathrm{L}$ & $\left\{R, T_{\text {high }}, \Delta T\right\}$ & $\begin{array}{c}\text { Adaptation } \\
\text { \{trials, a, ex, sweeps }\}\end{array}$ & $\{$ ex, mz, eq, sweeps, $m, \Delta m\}$ & $\mathrm{r}$ \\
\hline 1.0 & 8 & $\{21,4.7,0.05\}$ & $\{$ none $\}$ & $\{2000,1000,50,2,1,1\}$ & 100 \\
1.0 & 16 & $\{21,4.7,0.05\}$ & $\{$ none $\}$ & $\{2000,1000,70,3,1,1\}$ & 100 \\
1.0 & 32 & $\{11,4.7,0.1\}$ & $\{10,3,2000,10\}$ & $\{3000,1500,100,5,1,1\}$ & 500 \\
1.0 & 64 & $\{11,4.7,0.1\}$ & $\{58,2,2000,10\}$ & $\{5000,3000,200,5,1,1\}$ & 2000 \\
\hline
\end{tabular}

The physical observables are presented in Figure 11 and support the transition-like behavior at $T_{c} \sim 3.9$, or $\beta_{c} \sim 0.2631$, which was studied by Fytas and Malakis in their phase diagram [49]. The behavior can be observed in the specific heat curve as well as in susceptibility one, where the peaks of the curves tend to group at the region of temperature near $T_{c} \sim 3.9$ as $L$ increases. It is important to mention however that Malakis and Fytas have identified a lack of self-averaging for the specific heat in the 3D bimodal RFIM with $h_{i}= \pm 2$ [50]. The authors mention that averaging on the individual maxima can lead to better numerical results for this quantity. For the correlation length plot, which is expressed as $\xi / L$, the values obtained suggest that $\xi$ is rounded off to be in the order of linear size $L$ at the critical point, as predicted by the standard finite-size scaling theory. In the Binder plot, a transition-like behavior is observed where the curves take different paths, supporting the results by Fytas and Malakis [49].

The error bars correspond mostly to sample-to-sample fluctuations, which dominate the statistical error. For visual clarity, we only included the error bars of the largest size studied, i.e., $L=64$, nevertheless it is worth mentioning that the error bars for $L=8,16,32$ were even smaller than the ones for $L=64$.

\section{Technical considerations for $L=128$ and beyond}

Fytas et. al. have shown that the bimodal RFIM suffers from strong scaling corrections [51]. Because of this, there is a real interest on studying even larger sizes. It is technically possible to go beyond $L=64$ using the computational strategy proposed in this work, but a more important question is whether it is feasible or not. Based on preliminary simulations we have done, we expect that simulating $L=128$ with our hardware resources (two GPUs) would require at least three months, which we consider is on the limit of a practical computation for a physical research. Assuming the performance of actual GPUs such as the Tesla K40, we 

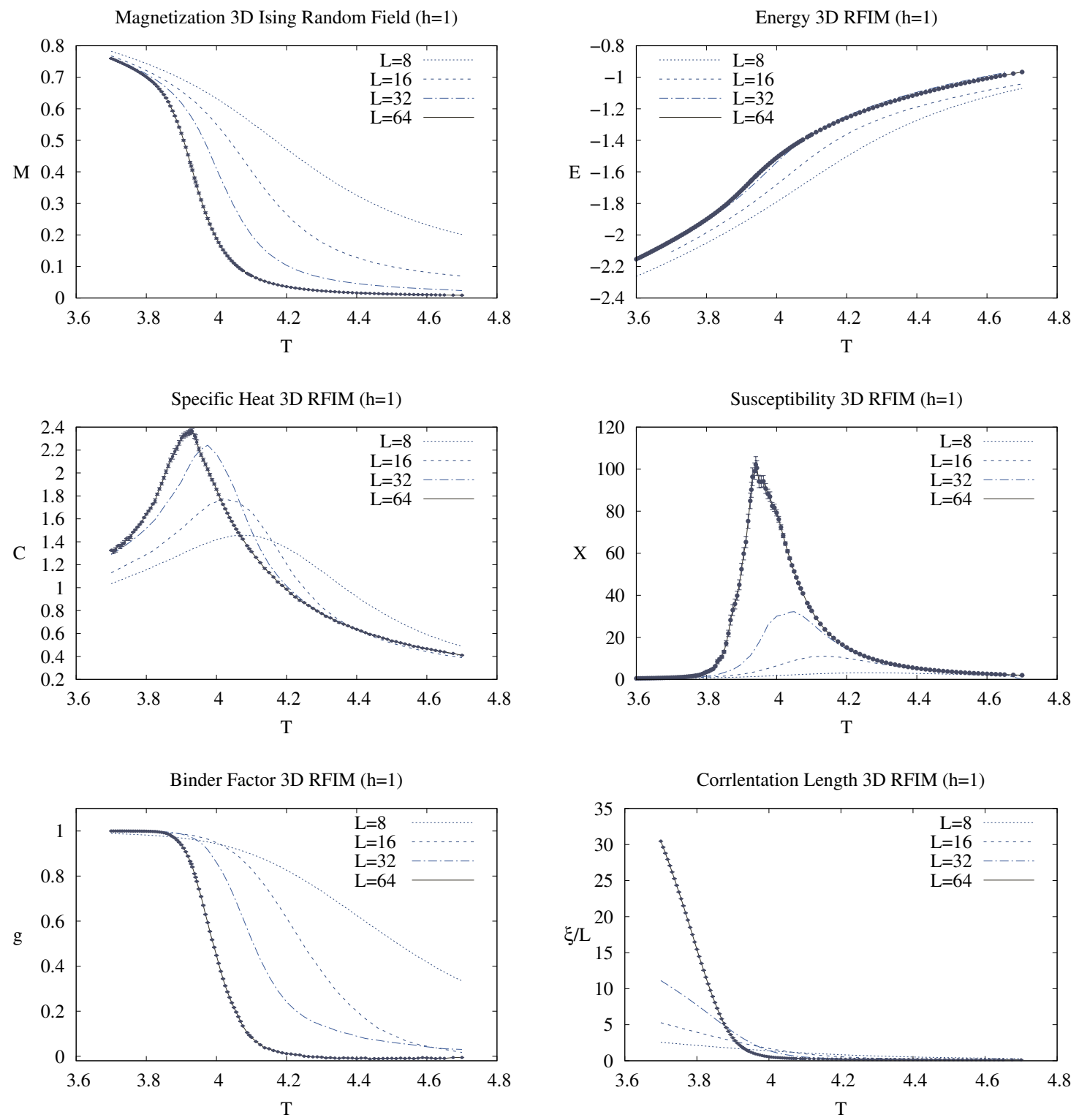

Figure 11: Preliminary physical observables for the 3D Random Field Ising Model with $h=1$.

can expect that around 16 GPUs for $L=128,180$ GPUs for $L=256$ and around 2048 GPUs for $L=512$ would lead to comfortable simulation times of a month or less. Given that GPU architectures improve each one or two years, one should expect a decrease on these numbers. In general, each time $L$ is doubled, we should provide at least eight times the computational power plus additional resources to cover the higher number of replicas required. We remind the reader that by increasing the number of GPUs together with $L$, one obtains almost $100 \%$ of efficiency, which is highly beneficial for large systems.

For the moment, physical results for $L=128$ could be eventually reached in the near future with medium scale hardware such as 16 or less GPUs. Sizes of $L=256,512$ would require a supercomputer and a much greater effort in order to achieve accurate results. For 
this last case our MPI implementation mpitrueke could be of help for the community.

\section{Discussion}

This work presented relevant computational details for a multi-GPU approach on the Exchange Monte Carlo method. The implementation is named trueke and is available to the community for the simulation of the 3D Random Field Ising Model. The parallelization strategy is organized in two levels; (1) spin-level parallelism, which scales in the presence of more cores and memory bandwidth per GPU, and (2) the replica-level parallelism that scales in the presence of additional GPUs. The spin-level parallelism is up to two orders of magnitude faster than its sequential CPU counterpart and one order of magnitude faster when compared to a multi-core CPU implementation running on a 16-core machine. When using $K>10$ multi-hit updates, the performance is very close to Weigel's highly optimized code for $2 \mathrm{D}$ Ising models. This aspect is important since it is yet to be studied if a moderate number of multi-hit updates, in the range $1 \leq K \leq 10$ can be useful for simulating the 3D RFIM efficiently without any loss of quality. If such possibility is true, then the simulation process can benefit from additional speedups, as in the case of continuous spin glasses [48].

The multi-GPU scaling showed that replica-level parallelism runs with over $95 \%$ of efficiency when ran on two GPUs. While the results are simple and probably predictable, projecting the performance to any number of GPUs is less trivial since the exchange part is considered in the performance measurement and it is done sequentially. It is important to answer the question how much impact does the sequential computation produce on the efficiency of the multi-GPU replica-level parallelism. In theory, and based on the results for two GPUs, we expect that doing a sequential exchange of temperatures has little impact on parallel multi-GPU efficiency as long as $L$ increases along with the number of GPUs. This way, the amount of parallel work increases faster than the sequential work as there are parallel reductions involved that grow cubically in $L$, whereas the sequential exchange grows linearly in the number of replicas. If only the number of GPUs and replicas increase, then we expect a degradation of the multi-GPU efficiency that is typical of strong scaling scenarios.

The adaptive strategy for selecting the temperature set was based on the idea of inserting new temperatures in between the lowest exchange rates found by an arbitrary number of trial runs. As a result, the simulation used more replicas at the temperature regions were exchange rates were originally low, and less replicas were the exchange rate was already good, such as in the high temperature regime. The adaptive strategy outperformed any homogeneous approach, since these last ones had to deal with an over-population of replicas at places that actually did not require more temperature points, resulting in extra computational cost and slower performance, and an under-population of replicas at the low temperature regime near $T_{c}$, generating low exchange rates. The adaptive method works better when a small number of points are added at each trial run, i.e., between one to five insertions at each trial run, because this way one focuses temperature insertions on the most critical regions. Compared to the method by Katzgraber et. al. [37], our adaptive method differs since it feedbacks from the local exchanges of pairs of temperatures, always lifting the minimum values observed by inserting new temperatures, while in the work of Katzgraber et. al. they feedback by counting the number of times a replica travels the whole temperature range and based on this information they move the temperature set. In the method by Bittner et. al [38], they also vary the number of Metropolis sweeps based on the auto-correlation times in order to

avoid two replicas getting trapped exchanging together, which they identify as a problem for 
Katzgraber method. The idea of not fixing the number of sweeps in the parallel tempering simulation, but instead choose the number dynamically based on a certain parameter is indeed promising for future optimizations. Nevertheless, inserting new temperatures provided this work the advantage that it simulates a subset of replicas, spending less computational time as compared to using the full set of replicas. Additionally, the insertions are local and do not compromise the rest of the temperature set. The computational technique proved to be simple yet very effective, since it is just based on an iterative approach that fills the $k$ minimum exchange rates observed at each trial run. As a result, it provides a distribution of temperature points that resembles a recursive divide and conquer approach.

The main reason for choosing multi-GPU computing at the replica-level, and not at the realization level (fully independent parallelism) as one would naturally choose, is mostly because the latter strategy is not prepared for the study of large lattice systems, which would be of great interest for the near future. From our experience with the 3D RFIM, the number of replicas needed to keep at least $20 \% \sim 40 \%$ of exchange rate grew very fast as $L$ increased. Thus, distributing the replicas dynamically across several GPUs extends the possibilities of studying larger disordered lattices. Nevertheless, the MPI version of trueke, mpitrueke, uses a third parallelization level for the different nodes, which is done at the disorder realization level.

The multi-GPU approach proposed, named trueke, has allowed us to study th 3D RFIM at size $L=64$ using $h=1$, for which its results can be useful as reference for future physical contributions to the field. It is technically possible to study more complex systems at $L>64$ and $h>1$. Although an increase in $h$ makes the physical simulation harder to reach a proper equilibrium and doubling $L$ makes the computational cost at least eight times higher, the parallel efficiency of a GPU-based method would not be compromised. Obtaining such physical results was out of the scope of this work, but it is an important aspect to consider as a future work as well as using new measuring techniques as the one proposed by Malakis and Fytas [50]. The implementations trueke and mpitrueke are available to the community as a tool or reference for studying such complex systems, specially for groups that dispose of many GPUs.

\section{Acknowledgements}

The authors would like to thank Prof. M. Weigel for his valuable feedback and explanations on the subject. Also, we greatly thank the Nvidia GPU Research Center at the Department of Computer Science (DCC) of University of Chile for supporting our research and giving us access to GPU hardware. This research is supported by FONDECYT project $N^{o} 3160182$ CONICYT, Chile; the National Science Foundation of China (NSFC) under Grant No. 11275185 and by the Open Project Program of State Key Laboratory of Theoretical Physics, Institute of Theoretical Physics, Chinese Academy of Sciences, China (No. Y5KF191CJ1).

\section{References}

[1] E. Ising, Beitrag zur theorie des ferromagnetismus, Zeitschrift Für Physik 31 (1) (1925) 253-258.

[2] B. A. Cipra, An introduction to the Ising model, Am. Math. Monthly 94 (1987) 937-959. doi:10.2307/2322600. 
[3] R. B. Potts, Some generalized order-disorder transformations, Cambridge Philos. Soc. Math. Proc. 48 (1952) 106-109.

[4] N. Metropolis, A. Rosenbluth, M. Rosenbluth, A. Teller, E. Teller, Equation of state calculations by fast computing machines, J. Chem. Phys. 21 (1953) 1087.

[5] W. K. Hastings, Monte Carlo sampling methods using markov chains and their applications, Biometrika 57 (1) (1970) 97-109. doi:10.1093/biomet/57.1.97.

[6] R. H. Swendsen, J.-S. Wang, Nonuniversal critical dynamics in Monte Carlo simulations, Phys. Rev. Lett. 58 (1987) 86-88.

[7] U. Wolff, Collective Monte Carlo updating for spin systems, Phys. Rev. Lett. 62 (1989) $361-364$.

[8] C. F. Baillie, P. D. Coddington, Comparison of cluster algorithms for two-dimensional Potts models, Phys. Rev. B 43 (1991) 10617-10621. doi:10.1103/PhysRevB.43.10617.

[9] N. Prokof'ev, B. Svistunov, Worm Algorithms for Classical Statistical Models, Physical Review Letters 87 (16) (2001) 160601+.

[10] A. D. Sokal, Overcoming critical slowing-down: Where do we stand 23 years after Swendsen and Wang?, Rutgers University (2010).

[11] A. P. Young, M. Nauenberg, Quasicritical behavior and first-order transition in the $d=3$ random-field ising model, Phys. Rev. Lett. 54 (1985) 2429-2432. doi:10.1103/PhysRevLett.54.2429.

URL http://link.aps.org/doi/10.1103/PhysRevLett.54.2429

[12] M. Gofman, J. Adler, A. Aharony, A. B. Harris, M. Schwartz, Critical behavior of the random-field ising model, Physical Review B 53 (10) (1996) 6362.

[13] P. Theodorakis, N. Fytas, Random-field ising model: Insight from zero-temperature simulations, arXiv preprint arXiv:1501.02338.

[14] Y. Imry, S.-k. Ma, Random-field instability of the ordered state of continuous symmetry, Phys. Rev. Lett. 35 (1975) 1399-1401. doi:10.1103/PhysRevLett.35.1399.

URL http://link.aps.org/doi/10.1103/PhysRevLett.35.1399

[15] K. Hukushima, K. Nemoto, Exchange Monte Carlo method and application to spin glass simulations, Journal of the Physical Society of Japan 65 (6) (1996) 1604-1608. doi:10.1143/JPSJ.65.1604.

[16] R. H. Swendsen, J.-S. Wang, Replica Monte Carlo simulation of spin-glasses, Phys. Rev. Lett. 57 (1986) 2607-2609. doi:10.1103/PhysRevLett.57.2607.

[17] C. Geyer, Markov Chain Monte Carlo maximum likelihood, in: Proceedings of the 23rd Symposium on the Interface, 1991, pp. 156-163.

[18] S. F. Edwards, P. W. Anderson, Theory of spin glasses, Journal of Physics F: Metal Physics 5 (5) (1975) 965. 
[19] A. T. Ogielski, I. Morgenstern, Critical behavior of three-dimensional Ising spin-glass model, Physical Review Letters 54 (9) (1985) 928.

[20] H. G. Katzgraber, M. Körner, A. Young, Universality in three-dimensional Ising spin glasses: A Monte Carlo study, Physical Review B 73 (22) (2006) 224432.

[21] H. Rieger, L. Santen, U. Blasum, M. Diehl, M. Jünger, G. Rinaldi, The critical exponents of the two-dimensional Ising spin glass revisited: exact ground-state calculations and Monte Carlo simulations, Journal of Physics A: Mathematical and General 29 (14) (1996) 3939.

[22] C. A. Navarro, N. Hitschfeld-Kahler, L. Mateu, A survey on parallel computing and its applications in data-parallel problems using GPU architectures, Commun. Comput. Phys. 15 (2014) 285-329.

[23] E. E. Ferrero, J. P. De Francesco, N. Wolovick, S. A. Cannas, q-state Potts model metastability study using optimized GPU-based Monte Carlo algorithms, Computer Physics Communications 183 (8) (2012) 1578-1587.

[24] T. Preis, P. Virnau, W. Paul, J. J. Schneider, GPU accelerated Monte Carlo simulation of the 2d and 3d ising model, Journal of Computational Physics 228 (12) (2009) 4468 4477. doi:http://dx.doi.org/10.1016/j.jcp.2009.03.018.

[25] B. Block, P. Virnau, T. Preis, Multi-GPU accelerated multi-spin Monte Carlo simulations of the 2d ising model, Computer Physics Communications 181 (9) (2010) 1549 - 1556. doi:http://dx.doi.org/10.1016/j.cpc.2010.05.005.

[26] M. Lulli, M. Bernaschi, G. Parisi, Highly optimized simulations on single- and multi-gpu systems of the 3d ising spin glass model, Computer Physics Communications 196 (2015) 290-303.

[27] M. Weigel, Simulating spin models on GPU, Computer Physics Communications 182 (9) (2011) 1833-1836.

[28] M. Weigel, Performance potential for simulating spin models on GPU, Journal of Computational Physics 231 (8) (2012) 3064-3082.

[29] M. Weigel, Simulating spin models on GPU: A tour, International Journal of Modern Physics C 23 (08).

[30] Y. Komura, Y. Okabe, Multi-GPU-based Swendsen-Wang multi-cluster algorithm for the simulation of two-dimensional q-state Potts model, Computer Physics Communications 184 (1) (2013) 40 - 44. doi:10.1016/j.cpc.2012.08.006.

[31] Y. Komura, Y. Okabe, GPU-based single-cluster algorithm for the simulation of the ising model, J. Comput. Phys. 231 (4) (2012) 1209-1215. doi:10.1016/j.jcp.2011.09.029.

[32] Y. Komura, Y. Okabe, Gpu-based Swendsen-wang multi-cluster algorithm for the simulation of two-dimensional classical spin systems, Computer Physics Communications 183 (6) (2012) 1155-1161. 
[33] M. Weigel, Connected-component identification and cluster update on graphics processing units, Phys. Rev. E 84 (2011) 036709.

[34] K. A. Hawick, A. Leist, D. P. Playne, Parallel graph component labelling with GPUs and CUDA, Parallel Comput. 36 (12) (2010) 655-678. doi:10.1016/j.parco.2010.07.002.

[35] Y. Delgado, A GPU-accelerated worm algorithm, Tech. rep., University of Grazz, Graz, Austria (2011).

[36] Y. Fang, S. Feng, K.-M. Tam, Z. Yun, J. Moreno, J. Ramanujam, M. Jarrell, Parallel tempering simulation of the three-dimensional edwards-anderson model with compact asynchronous multispin coding on GPU, Computer Physics Communications 185 (10) (2014) 2467 - 2478. doi:http://dx.doi.org/10.1016/j.cpc.2014.05.020.

[37] H. G. Katzgraber, S. Trebst, D. A. Huse, M. Troyer, Feedback-optimized parallel tempering Monte Carlo, Journal of Statistical Mechanics: Theory and Experiment 2006 (03) (2006) P03018.

[38] E. Bittner, A. Nußbaumer, W. Janke, Make life simple: Unleash the full power of the parallel tempering algorithm, Phys. Rev. Lett. 101 (2008) 130603. doi:10.1103/PhysRevLett.101.130603.

[39] I. T. P754, ANSI IEEE 754-1985 Standard for Binary Floating-Point Arithmetic, IEEE New York (Aug 12 1985).

[40] M. R. O’Neill, PCG: A Family of Simple Fast Space-Efficient Statistically Good Algorithms for Random Number Generation (2015).

URL http: / /www.pcg-random.org/paper.html

[41] NVIDIA, CUDA CURAND Library, NVIDIA Corporation, Santa Clara, CA, USA (2015).

[42] A. K. Hartmann, H. Rieger, Optimization Algorithms in Physics, 1st Edition, Wiley$\mathrm{VCH}$.

[43] Nvidia-Corporation, Nvidia CUDA C Programming Guide (2016).

[44] A. Kone, D. A. Kofke, Selection of temperature intervals for parallel-tempering simulations, The Journal of chemical physics 122 (20) (2005) 206101.

[45] N. Rathore, M. Chopra, J. J. de Pablo, Optimal allocation of replicas in parallel tempering simulations, The Journal of chemical physics 122 (2) (2005) 024111.

[46] B. Chapman, G. Jost, R. v. d. Pas, Using OpenMP: Portable Shared Memory Parallel Programming (Scientific and Engineering Computation), The MIT Press, 2007.

[47] J. Salas, A. D. Sokal, Universal amplitude ratios in the critical two-dimensional ising model on a torus, Journal of Statistical Physics 98 (3) 551-588.

[48] T. Yavors'kii, M. Weigel, Optimized GPU simulation of continuous-spin glass models, Eur. Phys. J. Spec. Top. 210 (2012) 159. 
[49] N. G. Fytas, A. Malakis, Phase diagram of the 3d bimodal random-field ising model, The European Physical Journal B 61 (1) (2008) 111-120. doi:10.1140/epjb/e2008-00039-7.

[50] A. Malakis, N. G. Fytas, Lack of self-averaging of the specific heat in the three-dimensional random-field ising model, Phys. Rev. E 73 (2006) 016109. doi:10.1103/PhysRevE.73.016109.

URL http://link.aps.org/doi/10.1103/PhysRevE.73.016109

[51] N. G. Fytas, V. Martín-Mayor, Universality in the three-dimensional random-field ising model, Phys. Rev. Lett. 110 (2013) 227201. doi:10.1103/PhysRevLett.110.227201. 